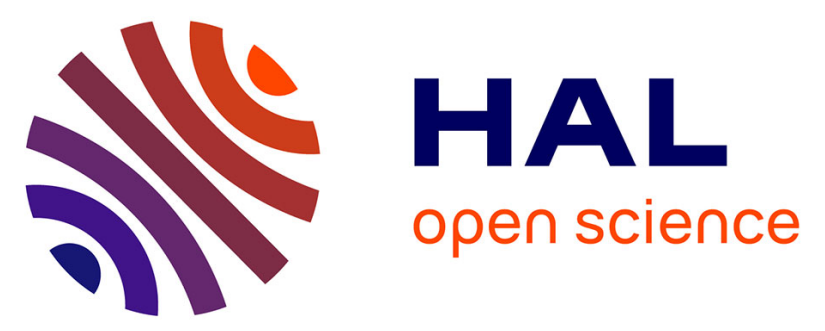

\title{
A game theory framework for a distributed and energy efficient bandwidth expansion process
}

\author{
Rodrigo-Alberto Vaca-Ramirez, John S. Thompson, Eitan Altman,
}

Victor-Manuel Ramos-Ramos

\section{> To cite this version:}

Rodrigo-Alberto Vaca-Ramirez, John S. Thompson, Eitan Altman, Victor-Manuel Ramos-Ramos. A game theory framework for a distributed and energy efficient bandwidth expansion process. 1st IEEE INFOCOM International Workshop on Green Cognitive Communications and Computer Networking (GCCCN 2014), Apr 2014, Toronto, Canada. pp.712 - 717, 10.1109/INFCOMW.2014.6849318 . hal01101217

\section{HAL Id: hal-01101217 \\ https://hal.inria.fr/hal-01101217}

Submitted on 8 Jan 2015

HAL is a multi-disciplinary open access archive for the deposit and dissemination of scientific research documents, whether they are published or not. The documents may come from teaching and research institutions in France or abroad, or from public or private research centers.
L'archive ouverte pluridisciplinaire HAL, est destinée au dépôt et à la diffusion de documents scientifiques de niveau recherche, publiés ou non, émanant des établissements d'enseignement et de recherche français ou étrangers, des laboratoires publics ou privés. 


\title{
A Game Theory Framework for a Distributed and Energy Efficient Bandwidth Expansion Process
}

\author{
Rodrigo A. Vaca Ramírez ${ }^{(1)}$, John S. Thompson ${ }^{(1)}$, Eitan Altman $^{(2)}$, and Víctor M. Ramos R. ${ }^{(3)}$ \\ ${ }^{(1)}$ The University of Edinburgh, Institute for Digital Communications \\ King's Buildings, Mayfield Road, Edinburgh, EH9 3JL, UK \\ ${ }^{(2)}$ INRIA, Sophia Antipolis Cedex, BP93, 06902 France \\ (3) Universidad Autónoma Metropolitana (UAM) \\ Department of Electrical Engineering Iztapalapa, Mexico City \\ E-Mail: \{r.vaca, john.thompson\}@ed.ac.uk, eitan.altman@inria.fr, vicman@xanum.uam.mx
}

Invited Paper

\begin{abstract}
In this paper, we aim to reduce the power expenditure in the reverse link during low network load periods, by allocating extra resource blocks (RBs) to the mobile users. Thereby, the user's rate demands are split among its allocated RBs in order to transmit in each of them by using a low level modulation order. We model the bandwidth expansion (BE) process by a game theory framework derived from the concept of stable marriage with incomplete lists (SMI). We focus on the optimization of the circuit consumed power rather than on the transmitted power. Thus, the power consumption of the radio frequency (RF) circuits and the base band (BB) system module is taken into account. Our proposed framework works in a distributed way, hence each RB and mobile user in the system are considered as an independent entity which takes an active role in the decision making process. Additionally, we show that when circuit power consumption is used as an optimization metric, transmitting in more than one RB may not become an energy efficient solution for users experiencing favorable propagation conditions in the reverse link.
\end{abstract}

\section{INTRODUCTION}

Wireless cellular communication systems have experienced a rapid development in recent years. This is due to the tenfold increase of data volume every five years which comes together with a doubling in energy consumption over the same period. Thus, academia and industry researchers have started to consider solutions to reduce the energy expenditure rather than only improving system's capacity.

Significant research has been performed in the context of green radio. In [1], the authors describe the most promising research directions to improve energy efficiency in wireless networks. Radio resource management techniques such as: interference mitigation and resource block (RB) allocation are proposed as an efficient way to reduce power consumption in communication systems. In [2], we propose a scheme for intercell interference reduction which trades capacity for energy efficiency. Capacity is reduced for users at the cell center which consequently reduces interference for users close to the cell edge. Thus, significant gains in energy efficiency can be obtained with a minimal reduction in system's throughput. An energy efficient RB allocation scheme, which is coordinated at the base station (BS) side is proposed in [3]. The authors show that by allocating extra RBs to the mobile users it is possible to reduce the transmitted power expenditure in the downlink while maintaining a constant data rate. In addition, the authors show that increasing the number of allocated RBs always provides an increase in the energy efficiency metric for the system. Nevertheless, their approach is only based in optimizing the transmitted power, hence the power expenditure of power amplifiers and the signal processing module is ignored. Moreover, the design and implementation of centralized approaches such as the one proposed in [3] may become an ambitious task due to the large scale nature of wireless systems [4]. This is because the complexity and implementation cost of centralized schemes tend to be a limiting factor in the network performance [2]. In [4], the authors use the game theory concept of Nash bargaining, in order to effectively assign sub-carriers to mobile users in a single cell scenario. They aim to maximize the overall system rate under power and rate constraints for each user. Moreover, they show that by using game theory, the system's complexity may be reduced while obtaining a similar performance to the centralized schemes.

Our main contribution in this paper is to model an energy efficient RB allocation process by using a low complexity distributed approach derived from the concept of stable marriage with incomplete lists (SMI) [5]. We focus on the optimization of the circuit consumed power rather than the transmitted power. In addition, we show that adding extra RBs results in a more energy efficient solution for users close to cell edge than for users close to the cell center. The remainder of this paper is as follows, in Section II we present the problem scenario and performance metrics. An analysis of the consequences of the proposed bandwidth expansion method in terms of energy efficiency are discussed in Section III. In Section IV, we describe our bandwidth expansion framework. A summary of the comparison schemes and the simulation scenario are described in Section V. Results are presented in Section VI. Finally, Section VII offers concluding remarks. 


\section{System Scenario and Performance Metrics}

We consider $K$ single antenna mobile stations (MSs) sending data on the uplink to a multiple antenna base station (BS). The system bandwidth $B(\mathrm{~Hz})$ is divided into $R$ resource blocks (RBs). Hence, an orthogonal frequency division multiple access (OFDMA) system is constructed. When the network is fully loaded, a single RB is assigned to each mobile user. However, during low load periods spare RBs are available, thus our proposed bandwidth expansion mechanism may be applied by allocating extra RBs to each mobile user.

\section{A. Channel Model}

1) Reverse Link: As mentioned above, the frequency selective channel is decomposed into $R$ RBs. Thus, the uplink received signal at the base station (BS) separated by a distance $d$ from a $k$-th MS is determined by path loss, log-normal shadowing, and channel variations caused by frequency selective fading. The channel exhibits Rayleigh fading, whose power delay profile has been taken from [6]. Path loss and shadowing are modeled as a power fall off that subsequently attenuates the power of the transmitted signal. Thus, the average power of the received signal on the $r$-th RB can be expressed by [2]:

$$
P r_{k}^{r}=P t_{k}^{r} 10^{\frac{-L\left(d_{k}\right)+X_{\sigma}}{10}},
$$

where $P t_{k}^{r}$ denotes the signal transmitted power the $k$-th user in the $r$-th RB, $X_{\sigma}$ is the log-normal shadowing value $(\mathrm{dB})$ with standard deviation $\sigma$, and $L\left(d_{k}\right)$ is the distance-dependent path loss $(\mathrm{dB})$, which is calculated as follows:

$$
L\left(d_{k}\right)=15.3+37.6 \log _{10}\left(d_{k}\right) .
$$

We assume the channel is know at the transmitter and receiver. Therefore, the received signal at the BS side on the $r$-th $\mathrm{RB}$ for the $k$-th user is given by:

$$
\mathbf{y}_{k}^{r}=\sqrt{\operatorname{Pr}_{k}^{r}} \mathbf{h}_{k}^{r} s_{k}^{r}+\mathbf{n}_{k}^{r},
$$

where $\mathbf{h}_{k}^{r}$ is the $1 \times M_{r}$ channel matrix on the $r$-th $\mathrm{RB}, s_{k}^{r}$ is the information symbol with unit energy and $\mathbf{n}_{k}^{r}$ is the $M_{r} \times 1$ noise factor. Hence, the received signal noise ratio (SNR) at the BS side in the $r$-th RB may be expressed by [7]:

$$
\eta_{k}^{r}=\frac{\left\|\mathbf{h}_{k}^{r}\right\|_{F}^{2} \operatorname{Pr}_{k}^{r}}{\rho}
$$

where $\rho$ is the noise power. In addition, by combining Eqs. (1) and (4) and by making $G_{k}^{r}=\left\|\mathbf{h}_{k}^{r}\right\|_{F}^{2} 10^{\frac{-L\left(d_{k}\right)+X_{\sigma}}{10}}$. Eq. (4) may be written as follows:

$$
\eta_{k}^{r}=\frac{P t_{k}^{r} G_{k}^{r}}{\rho}
$$

where $G_{k}^{r}$ represents the channel path-gain between the BS and the $k$-th MS in the $r$-th RB.

\section{B. Power Consumption Model}

In this work, we take into account the circuit power expenditure caused by information transmission in the uplink. The uplink power expenditure mainly depends on components such as the base-band (BB) module and the radio frequency (RF) module. The former encloses the power expenditure due to to the base-band signal processing (e.g., channel coding and decoding), and the latter includes the power consumption of the radio frequency parts (e.g., the power amplifier). Therefore, to model the power consumption of both modules, we use the model proposed in [8], where an analysis of the power consumption for both modules in a Long Term Evolution (LTE) MS is presented.

In order to model the circuit consumed power of the RF module a power amplifier array is assumed $[8,9]$. The power amplifier array is built based on a $10[\mathrm{dBm}]$ low power amplifier (LPA) and two high power amplifiers (HPA); HPA 1, and HPA 2, where the power amplifier efficiency is assumed equal for both high power amplifiers. However, HPA 2 is designed to transmit at $20[\mathrm{dBm}]$, which is half of the maximum transmitted power of HPA 1 . Therefore, the circuit power consumption in the uplink $P_{k}^{r}\left(P t_{k}^{r *}\right)[\mathrm{W}]$ may be represented as follows.

$$
P_{k}^{r}\left(P t_{k}^{r *}\right)= \begin{cases}2+0.005\left(P t_{k}^{r *}\right)-A & 10 \geq P t_{k}^{r *} \\ \frac{1.2+0.12\left(P t_{k}^{r *}\right)-\left(A-P_{B B}\right)}{2} & 20 \geq P t_{k}^{r *}>10 \\ 1.2+0.12\left(P t_{k}^{r *}\right)-A & 23 \geq P t_{k}^{r *}>20\end{cases}
$$

where the $P t_{k}^{r *}$ is the transmitted power value $P t_{k}^{r}$ in [dBm], and $A$ comprises a set of constant values defined as follows [8]:

$$
A=P_{T x}+P_{\text {con }}-P_{B B}[W],
$$

where $P_{T x}$ is the base power that the RF chain consumes in transmission mode, $P_{\text {con }}$ is the MS's power consumption when connected to the $\mathrm{BS}$, and $P_{B B}$ is the power consumed by the $\mathrm{BB}$ module [8].

\section{Performance Metrics}

The throughput between the $k$-th MS and the BS in the $r$-th $\mathrm{RB}$ using adaptive modulation and coding is computed by [2]:

$$
T_{k}^{r}\left(\eta_{k}^{r}\right)=k_{s c} \varrho_{s} \varepsilon\left(\eta_{k}^{r}\right)[\mathrm{bits} / \mathrm{s}],
$$

where $k_{s c}$ is the number of subcarriers per resource block, $\varrho_{s}$ is the symbol rate per subcarrier, and $\varepsilon\left(\eta_{k}^{r}\right)$ is the spectral efficiency for a Long Term Evolution (LTE) system [2].

Moreover, the total user throughput for the $k$-th user can be obtained by:

$$
T_{k}=\sum_{r=1}^{z} T_{k}^{r}[\text { bits } / \mathrm{s}],
$$

where $z$ is the bandwidth expansion factor, which represents the number of RBs allocated to the $k$-th MS. Additionally, the 
total user consumed power for the $k$-th MS is represented by:

$$
P_{\text {total_k }}=\sum_{r=1}^{z} P_{k}^{r}\left(P t_{k}^{r *}\right)[W] .
$$

The user energy efficiency $\beta_{k}$ measures the user throughput per unit of consumed power.

$$
\beta_{k}=T_{k} / P_{\text {total_k }}[\text { bits } / \mathrm{J}] \text {. }
$$

Furthermore, the system energy efficiency $\beta_{\text {sys }}$ describes the correspondence between the total user throughput and the total power spent by all the users in the system.

$$
\beta_{\text {sys }}=\sum_{k=1}^{K} T_{k} / \sum_{k=1}^{K} P_{\text {total_k } k}[\text { bits } / \mathbf{J}] .
$$

where $K$ is the number of total users in the system.

\section{THEORETICAL ANALYSIS}

In order to show the improvements in energy efficiency, Shannon's capacity formula will be used for ease of analysis and without loss of generality. Thereby, the capacity in the $r$-th RB may be written as follows:

$$
C_{k}^{r}=B \log _{2}\left(1+\eta_{k}^{r}\right)
$$

where $B$ represents the $r$-th's RB bandwidth. Moreover, assume that for the bandwidth expansion (BE) scheme, we aim to have the same overall transmission rate after more $\mathrm{RBs}$ are allocated to the $k$-th user. Thus, this requirement may be represented by:

$$
z\left(B \log _{2}\left(1+\eta_{k_{-} \mathrm{BE}}^{r}\right)\right)=B \log _{2}\left(1+\eta_{k}^{r}\right),
$$

where $\eta_{k_{-} \mathrm{BE}}^{r}$ is the SNR of the $k$-th user in the $r$-th RB when the bandwidth expansion scheme is used. Recall that in an OFDMA system all the operations are always performed on an RB basis. Hence, by algebraic manipulations (14) becomes:

$$
\left(1+\eta_{k_{-} \mathrm{BE}}^{r}\right)^{z}=\left(1+\eta_{k}^{r}\right)
$$

By combining Eqs. (4) and (15), we may obtain the transmit power expenditure due to the BE scheme [3].

$$
P t_{k \_\mathrm{BE}}=\frac{\rho}{G_{k \_\mathrm{BE}}^{r}}\left(\left(1+\eta_{k}^{r}\right)^{1 / z}-1\right),
$$

The required transmitted power due to different values of $z$ is plotted in Fig. 1. The ratio $\frac{\rho}{G_{k-\mathrm{BE}}^{r}}$ is taken as constant value, under the assumption that the ${ }^{-\mathrm{BE}}$ noise that an $\mathrm{RB}$ experiences is not directly affected by allocating extra RBs, since all operations are performed on an per RB basis. Moreover, to improve system performance, the propagation conditions (e.g., frequency selective fading) of the new allocated RBs should be similar or better to the current allocated RB as we will show later in the paper.

In Fig. 1, we can see that increasing the number of RBs always provides transmission power savings, since a significant decrease in transmission power is observed as the value of $z$ increases. Nevertheless, in Fig. 2 we can be seen that this behavior does not remain the same when circuit power consumption is considered by including the model in (6). Adding extra RBs is not an energy efficient solution at low SNR levels (e.g., SNR $\leq 6[\mathrm{~dB}]$ ). Thus, transmitting with only one $\mathrm{RB}$ is the most energy efficient solution in this regime. This is because, in a low SNR regime a low required transmitted power is also required, thus the power circuit expenditure due to the $\mathrm{BB}$ and RF modules, when transmitting in more than one $\mathrm{RB}$, does not become and energy efficient solution in this instance. Moreover, we can see that $z=2$ tends to be the most energy efficient solution from 6 to 15 [dB]. Finally, using more than 2 RBs provides the most power savings for high SNR levels (e.g., SNR $\geq 15$ [dB] ). This is because, in a high SNR regime a high transmitted power is also required. Hence, the circuit power consumption for utilizing the RF and BB to transmit in multiple RBs is justified in this case.

Moreover, when channel effects are taken into account, it should be understood when is worthwhile to add extra RBs to a specific user. Since channel propagation conditions such as frequency selective fading may not remain the same within different RBs. Hence in terms of transmitted power, this requirement can be represented by [3]:

$$
P t_{k}^{r} \geq \sum_{r=1}^{z} P t_{k_{-} \mathrm{BE}}^{r} \rho .
$$

In terms of circuit consumed power the equation above becomes:

$$
P_{k}^{r}\left(P t_{k}^{r *}\right) \geq \sum_{r=1}^{z} P_{k_{-} \mathrm{BE}}^{r}\left(P t_{k_{-} \mathrm{BE}}^{r}\right)
$$
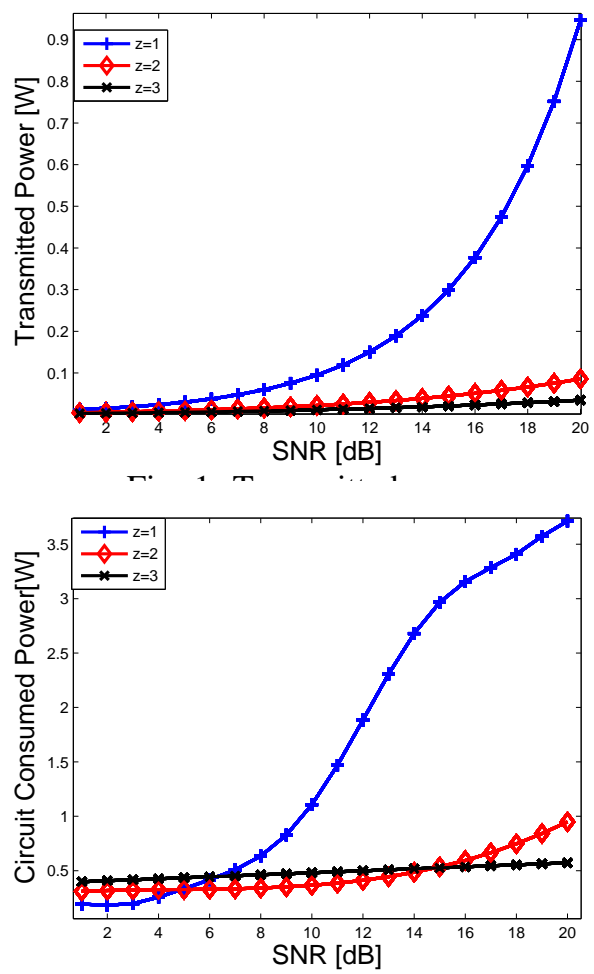

Fig. 2: Circuit power consumption. 


\section{Distributed BANDWIDTh EXPANSION FRAMEWORK}

In order to obtain energy savings, we model the bandwidth expansion process by a game theoretic approach derived from the concept of stable marriage with incomplete lists (SMI) [5]. The SMI framework is used to find a stable match between two sets (MSs and RBs). A match is said to be stable when no better alternative pairing exists in the other set compared with the currently matched elements. Thereby, the SMI reaches a stable solution for the BE process [5].

In our proposed framework, the set of spare RBs in the system consists in $F \mathrm{RBs}$, thus $F \in R$, and the other set represents the $K$ mobile stations. Moreover, in order to perform the bandwidth expansion process every element in each set should build a list which consists of the members of the opposite set ordered by preference. A matching pair $M$ is a tuple (MS, RB) such that each MS and RB belongs exactly to one tuple. If $(k, r) \in M$, we say that the $r$-th RB is the $k$-th MS's partner in $M$ and vice versa. Notice that when the SMI is used, the maximum value that the BE factor can take is $z=2$. This is because, the BE expansion by using the stable marriage only admits to allocate an extra RB per user. Nevertheless, SMI can still be an optimal approach since bandwidth is a limited resource in communications systems, thus the number of spare RBs in the network may always limited to an small number. The distributed BE procedure is modeled as follows:

1) First, the channel quality information is obtained at the BS side by Sounding Reference Signals (SRS) transmitted by the MS [10].

2) Further, this information may be forwarded to the MSs through the feedback channel. Thereby, MSs are able to compute the benefit of using the BE method by Eq. (18).

$$
U_{k r}=P_{k}^{r}\left(P t_{k}^{r *}\right)-\sum_{r=1}^{2} P_{k_{-} \mathrm{BE}}^{r}\left(P t_{k_{-} \mathrm{BE}}^{r}{ }^{*}\right),
$$

where Eq. (19) represents the difference in power expenditure when using a single RB for transmission compared with the $\mathrm{BE}$ method. Hence, the higher the utility value, the more willing is the $k$-th MS to use the $r$-th $\mathrm{RB}$ in the $\mathrm{BE}$ process. Moreover, if the utility becomes negative the MS will ignore the $r$-th $\mathrm{RB}$, since by choosing the $r$-th $\mathrm{RB}$, the power consumption in the uplink increases when the $\mathrm{BE}$ is used. Furthermore, the MS preference list $L_{k}$ is formed by evaluating each element of the subset $F$ with Eq. (19). MSs not obtaining any benefit from using the BE method with any of the RBs in the set, should transmit in a single RB.

3) In addition, the RBs need to compute the suitability for the BE process with a specific MS. Thus, each RB ranks the available MSs in the network as follows:

$$
U_{r k}=\sum_{j=1, j \neq k}^{N}{ }^{1} P_{k}^{r}\left(P t_{k}^{r *}\right) \leq P_{j}^{r}\left(P t_{j}^{r *}\right),
$$

where $U_{r k}$ is a utility function which consists of the pairwise comparisons of the $r$-th RB when allocated to the $k$-th MS against any other $j$-th MS in the network, where $j \in K$. The
Initialization: All MSs use a single RB for transmission; while There is an $R B$ willing to get into the $B E$ with a $k$-th $M S$;

do $M S_{h}$ is the highest MS in the $r$-th RB preference list, $L_{r}$, to whom it has not yet proposed; if $M S_{h}$ is free then the $r$-th RB and $M S_{h}$ become engaged; else

$M S_{h}$ is already engaged with a $j$-th RB (where $j \neq r$ );

if $M S_{h}$ prefers the $r$-th $R B$ to the $j$-th $R B$ in its preference list, $L_{k}$; then

$r$-th RB becomes engaged, and the $j$-th RB becomes free;

else

$M S_{h}$ remains engaged to the $j$-th RB; $M S_{h}$ is deleted from the list of the $r$-th RB, $L_{r}$

end

end

end

Algorithm 1: Stable Marriage with incomplete lists (SMI).

operator ${ }^{\mathbb{1}} A \leq B$ provides an output of 1 when the proposed condition holds, and 0 in any other case. Thus, a high utility value means that allocating the $r$-th RB to the $k$-th MS will contribute to higher energy savings in the system. Moreover, the RB's preference list, $L_{r}$, is formed by evaluating each $k$-th MS with Equation (20).

4) After the ranking is done, the SMI procedure given in Algorithm (1), can be applied.

\section{COMPARISOn Schemes And Simulation Scenario}

To evaluate the performance of our algorithm, we describe in this section three BE schemes which can allocate extra RBs to reduce the power expenditure in the reverse link. In addition, we implement a baseline scheme where all the MSs transmit in a single RB.

\section{A. Arbitrary RB allocation (ARBA)}

In this scheme, the extra RBs used by the $k$-th MS for transmission in the reverse link are chosen on a random basis.

\section{B. Stable marriage}

By using Algorithm (1), the BE expansion process through stable marriage can applied to increase the energy efficiency in the network.

\section{Centralized global optimum}

A centralized global optimum approach, which is based on an exhaustive search coordinated from the BS side, is implemented in order to find the price of anarchy for our proposed method. The price of anarchy is computed as the difference in performance when comparing a distributed with a centralized method. 
TABLE I: Simulation parameters.

\begin{tabular}{cc}
\hline \hline Parameter & Value \\
\hline MSs per macro-cell, $K$ & 20 \\
Number of antenas at the receiver, $M_{r}$ & 5 \\
Cell radius & $150 \mathrm{~m}$ \\
Number of available RBs, $R$ & 45 \\
Number of cells, $D$ & 1 \\
Subcarriers per RB, $k_{s c}$ & 12 \\
Symbol rate per subcarrier, $\varrho_{s}$ & $15 \mathrm{ksps}$ \\
$P_{T x}$ & $31.8 \mathrm{dBm}$ \\
$P_{\text {con }}$ & $23.8 \mathrm{dBm}$ \\
$P_{B B}$ for $\varepsilon=4.523$ bits & $11.9 \mathrm{dBm}$ \\
Maximum user transmit power & $23 \mathrm{dBm}$ \\
Shadowing, Std. Dev., $\sigma$ & $3 \mathrm{~dB}$ \\
Target SNR, & $17 \mathrm{~dB}$ \\
$\varepsilon$ for $17 \mathrm{~dB}$ SNR & $4.523 \frac{\text { bits }}{\text { symbol }}$ \\
$z$ & 2 \\
\hline
\end{tabular}

\section{Simulation scenario}

Monte Carlo simulations are performed using the parameters listed in Table I. This allows comparison of the performance of our method with the other schemes presented above. The simulation is comprised of a single cell with the mobile stations distributed uniformly over the cell. The cell is served by an omnidirectional BS. Furthermore, the system is thermal noise limited. We assume low network load conditions (e.g., $40 \%$ to $60 \%$ of the maximum capacity) thus spare RBs are available in the network. In our simulation, we assume that all the users in the network should achieve the same target SNR.

\section{RESUlTS}

In this section, we compare the performance in terms of energy efficiency and circuit power expenditure for the schemes presented in the last section. In Fig. 3, we plot the circuit power expenditure against different distances from the BS. We can observe that when MSs are close to the cell center, the user's power consumption of the centralized global optimum and SMI schemes overlaps with the baseline method. This is because mobile users are able to experience more favorable propagation conditions when close to the cell center. Thus as shown in Section III, the generated power cost due to the $\mathrm{BB}$ and RF modules when transmitting with more than

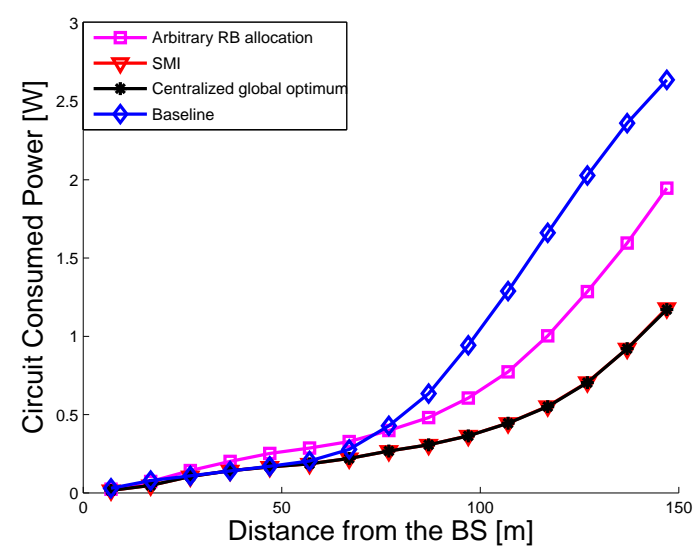

Fig. 3: User circuit consumed power vs distance from the BS.

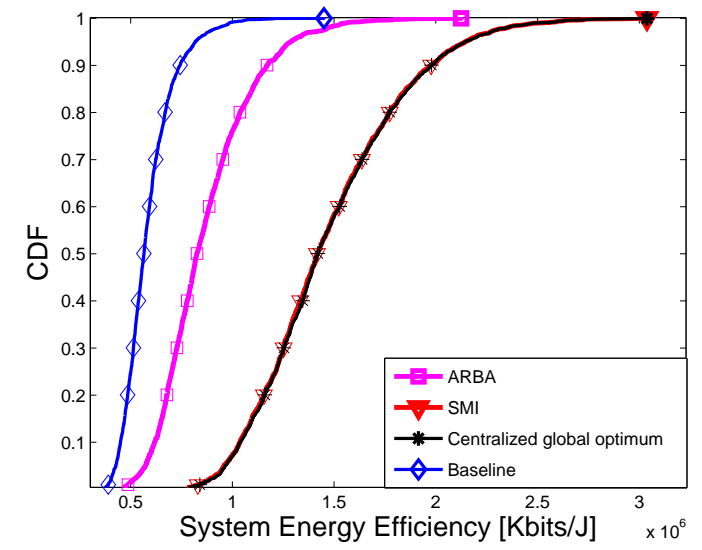

Fig. 4: System CDF energy efficiency.

one RB does not become an optimal solution in a low power tranmission regime. Moreover, since the SMI scheme performance is based on optimizing the utility function, Eq. (19), it allows our proposed algorithm to use more than one RB only when it generates significant power savings. In the case of the arbitrary RB allocation (ARBA) scheme, we can see how at close distances from the BS, this method consumes more power to achieve the same rate constraints than other approaches. This difference in performance is because, the ARBA may allocate more than one RB when transmitting at close distances from the BS. Furthermore, for users close to the cell edge (e.g., $140 \mathrm{~m}$ ), we can observe that less power is required when more than one RB are allocated to them. Since users that utilize the ARBA, the SMI and then centralized method required less circuit consumed power than the baseline method. Thus, it can be understood that in order to reduce energy consumption in the system, the extra RBs should be allocated mostly for users close to the edge rather than for cell center users.

Additionally in Fig. 4, we show the system energy efficiency. At the $50^{\text {th }}$ percentile, the SMI and the centralized method show performance improvements of $115 \%$ and $72 \%$ when compared to the baseline and ARBA, respectively. Hence, the proposed SMI approach is more energy efficient than the other two approaches. This gains in energy efficiency for the SMI and the centralized method compared to the other approaches are because both schemes allocates efficiently most of the RBs at the cell edge rather than at the cell center. Furthermore, notice that the performance of the centralized optimum method is the same as for the SMI scheme. Thus, for this case the price of anarchy of the proposed approach is zero.

To conclude our comparison, we discuss the complexity of the SMI and the centralized approach. It can be seen that the SMI method bases its optimization on Eqs (19) and (20), which induces a complexity of $(|F|)^{2}$ and $(K)^{2}$ respectively, where $|$.$| defines the cardinality of the subset. Furthermore,$ the bandwidth expansion scheme is based on the assumption of having more RBs available than MSs present in the system, thus the complexity of the system is bounded by 
the number of RBs rather than by the number of MSs. So, the complexity induced by the computation of the utility functions is $(|F|)^{2}$. Moreover, forming the preference lists $L_{k}$ and $L_{r}$ and performing the Algorithm (1) requires a sorting and a binary search operation respectively, thus a complexity of $F \log (|F|)$ and a complexity of $\log (|F|)$ is induced for each calculation. The dominant factor will be the one with the largest exponent, thus the complexity of the SMI is upper bounded by $(|F|)^{2}$. In the case of the centralized method, a exhaustive search approach when the number of elements is discrete, is considered $\mathcal{N} \mathcal{P}$-complete [11]. The total number of possible combinations can be computed by $\sum_{k=1}^{z-1}\left(\begin{array}{c}|F| \\ k\end{array}\right)$, where $\left(\begin{array}{c}|F| \\ k\end{array}\right)=\frac{|F| !}{k !(|F|-k) !}$. Moreover, each combination should be evaluated by Eq. (18), therefore a complexity of $\left(\sum_{k=1}^{z-1}\left(\begin{array}{c}|F| \\ k\end{array}\right)\right)^{2}$ is induced. For both methods complexity scales linearly with the number of mobiles in the system, $K$. The, order $\mathcal{O}$ notation is used to describe the growth rate of both schemes. Hence, the centralized method has a complexity of $\mathcal{O}\left(K \times\left(\sum_{k=1}^{z-1}\left(\begin{array}{c}|F| \\ k\end{array}\right)\right)^{2}\right)$ compared to a complexity of $\mathcal{O}\left(K \times|F|^{2}\right)$ for the SMI scheme. In case that $z \geq 2$, the complexity of the centralized method increases exponentially as $z$ increases. In the SMI case, it can be substituted by a more powerful scheme called the college admission framework, which is able to perform a BE for $z \geq 2$ while maintaining almost the same complexity of the SMI scheme [12].

\section{CONCLUSIONS}

In this work, we propose a distributed algorithm for bandwidth expansion derived from the SMI concept. In the proposed approach each RB and mobile user in the system are considered as an independent entity which takes an active role in the decision making process. From the results obtained, we showed that our approach is a more energy efficient solution with improvements of $115 \%$ and $72 \%$ when compared to the baseline and the ARBA scheme respectively. The performance of our method is the same in bits per Joule as the centralized global optimum, thus the price of anarchy in this case is zero. Furthermore, we have shown analytically and by simulation that when the mobile user experience favorable propagation conditions in the reverse link, the circuit consumed power is reduced by transmitting in one $\mathrm{RB}$ rather than using an $\mathrm{RB}$ expansion technique.

\section{REFERENCES}

[1] C. Han, T. Harrold, S. Armour, I. Krikidis, S. Videv, P. M. Grant, H. Haas, J. Thompson, I. Ku, C.-X. Wang, T. A. Le, M. Nakhai, J. Zhang, and L. Hanzo, "Green radio: radio techniques to enable energyefficient wireless networks," IEEE Communications Magazine, vol. 49, no. 6 , pp. $46-54,2011$.

[2] R. Vaca, J. Thompson, and V. Ramos, "Non-cooperative uplink interference protection framework for fair and energy efficient Orthogonal Frequency Division Multiple Access networks," IET Communications, vol. 7, no. 18, pp. 2015-2025, 2013.

[3] S. Videv and H. Haas, "Energy-efficient scheduling and bandwidthenergy efficiency trade-off with low load," in IEEE International Conference on Communications (ICC), 2011, pp. 1-5.
[4] Z. Han and R. Liu, "Fair multiuser channel allocation for OFDMA networks using Nash bargaining solutions and coalitions," IEEE Transactions on Communications, vol. 53, no. 8, pp. 1366-1376, 2005.

[5] K. Iwama, , and S. Miyazaki, "A survey of the stable marriage problem and its variants," in Proceedings of the International Conference on Informatics Research for Development of Knowledge Society Infrastructure, Jan 2008, pp. 131-136.

[6] A. Lozano and N. Jindal, "Transmit diversity vs. spatial multiplexing in modern MIMO systems," IEEE Transactions on Wireless Communications, vol. 9, no. 1, pp. 186-197, 2010.

[7] A. Paulraj, R. Nabar, and D. Gore, Introduction to space-time wireless communications, C. U. Press, Ed. Cambridge University Press, Jul. 2003.

[8] A. Jensen, M. Lauridsen, P. Mogensen, T. Srensen, and P. Jensen, "LTE UE power consumption model for system level energy and performance optimization," in Proceedings of the IEEE 76th Vehicular Technology Conference, Sep. 2012, pp. 1-6.

[9] K. Bobae, K. Cholho, and L. Jongsoo, "A dual-mode power amplifier with on-chip switch bias control circuits for LTE handsets," IEEE Transactions on Circuits and Systems, vol. 58, no. 12, pp. 857-861, 2011.

[10] S. Sesia, I. Toufik, and M. Bake, LTE-The UMTS Long Term Evolution From Theory to Practice, 1st ed., C. Wiley, Ed. Wiley, Chichester, 2009.

[11] Z. Han and K. J. R. Liu, Resource Allocation for Wireless Networks, C. U. Press, Ed. Cambridge University Press, Jul. 2008.

[12] D. Gale and L. S. Shapley, "Collegue admissions and the stability of marriage," The American Mathematical Monthly, vol. 69, no. 1, pp. 9 15, 1962. 